\title{
On the environmental decoherence and spin interference in mesoscopic loop structures
}

\author{
I.Tralle ${ }^{*}$ W. Paśko \\ Institute of Physics, University of Rzeszów \\ Al. T. Rejtana 16A, 35-310 Rzeszów, Poland
}

\begin{abstract}
Mechanisms of 'environmental decoherence' such as surface scattering, ElliotYafet process and precession mechanisms, as well as their influence on the spin phase relaxation are considered and compared. It is shown that the 'spin ballistic' regime is possible, when the phase relaxation length for the spin part of the wave function $\left(L_{\varphi}^{(s)}\right)$ is much greater than the phase relaxation length for the 'orbital part' $\left(L_{\varphi}^{(e)}\right)$. In the presence of an additional magnetic field, the spin part of the electron's wave function (WF) acquires a phase shift due to additional spin precession about that field. If the structure length $L$ is chosen to be $L_{\varphi}^{(s)}>L>L_{\varphi}^{(e)}$, it is possible to 'wash out' the quantum interference related to the phase coherence of the 'orbital part' of the WF, retaining at the same time that related to the phase coherence of the spin part and, hence, to reveal corresponding conductance oscillations.
\end{abstract}

PACS: 73.23.Ad, 75.47.Jn

Key words: Spin transport; Mesoscopic structures; Spin Coherence; Quantum interference; Spintronics

\section{Introduction}

One of the main ideas which underpins different possible applications of 'spin transport', including information storage and computation, is that the spins of electrons in semiconductors may have very long quantum coherence times [1,2], or in other words, electrons can travel a long way without flipping their spins. But this also gives the possibility to observe quantum effects which involve the interference of electron waves. In the classical picture of transport phenomena, the total probability for a particle to transfer from one point to another is the sum of the probabilities for such transfer over all possible trajectories. In the quantum description, this result corresponds to neglecting the interference of

\footnotetext{
*Corresponding author e-mail addresses: tralle@univ.rzeszow.pl
} 
scattered electron waves propagating along different paths. The destruction of quantum coherence is controlled by the phase relaxation time or phase relaxation length. Since for the electron spin this length may be very long, it is naturally to expect that the spin interference can reveal itself in the conductance oscillations similar to that ones which are due to Aharonov-Bohm effect [3]. Most of the researchers who dealt with the AharonovBohm effect considered mainly the Hamiltonian $\hat{H}=(\mathbf{p}-(\mathrm{e} / \mathrm{c}) \mathbf{A})^{2} / 2 \mathrm{~m}^{*}+\mathrm{U}(\mathrm{y}, \mathrm{z})$, where $\mathrm{U}(\mathrm{y}, \mathrm{z})$ is the energy corresponding to the transverse motion, and almost nobody takes into account the spin-part $\mu_{B} \hat{\sigma} \mathbf{B}$ of the Hamiltonian $\left(\mu_{B}\right.$ is the Bohr magneton, $\hat{\sigma}$ is the electron spin operator, $\mathbf{B}$ - magnetic field). The reason for the neglecting of this part of the Hamiltonian is that one usually treats electron's spin as something which is of no importance for the transport properties (for instance, current) of solids, semiconductors in particular. However, if the quantum interference is concerned, it might happen that the spin of electrons can play an important role $[1,2,4]$. If one considers the total Hamiltonian which includes Pauli term, one inclines (being in the framework of Pauli's spin theory) to treat $\sigma= \pm \hbar / 2$ as 'the projection of spin vector onto quantization axis' which coincide with external magnetic field B. But everybody who ever dealt with Dirac equation knows pretty well that it is not the case. May be it is worth-while to quote here the words of one of the most influential contemporary mathematicians, Yu. Manin, who wrote [5]: ' ... we only mislead ourselves if we awkwardly try to describe the internal quantum degrees of freedom as the 'value of projection of spin on the $z$-axis', since a spin vector lies in a completely different space from the $z$-axis'. Nevertheless, this naïve treatment of $\sigma$ as the 'projection of spin vector onto z-axis', to paraphrase John Bell's words, is sufficient for almost all practical purposes. Moreover, it can be easily amended by taking into account the true structure of corresponding Hilbert space and simply by writing down the electron wave function in factorized form as the direct product: $\Psi(\mathbf{r}, s)=\varphi(\mathbf{r}) \otimes \chi(s)$. It is worthy to note also, that if the quantum interference is concerned, the quantity of main importance is the phase coherence length. Introducing the factorized form of the wave function, one not only can, but rather have to introduce simultaneously two phase relaxation lengths, the first one for the 'orbital part' of the electron wave function, $L_{\varphi}^{(e)}$, and the second one, $L_{\varphi}^{(s)}$ for the spin part. It turns out [4], that $L_{\varphi}^{(s)}>>L_{\varphi}^{(e)}$, which is in total agreement with the experiment $[1,2,6]$. The physics which is behind that, is the following. An electron during its transfer along some path in the solid (semiconductor, for definiteness) interacts all the time with the environment. As a rule rigid scatterers such as impurities and other defects of crystalline structure, do not contribute to the phase relaxation; only dynamical scatterers like phonons, do. On the other hand, the electron scattering by phonons is mainly inelastic, while impurity scattering is mainly elastic, so we can say that only inelastic scattering contributes to the phase relaxation. But what does it mean inelastic scattering in case of spin? It means spin flips which are the consequences of scattering by phonons accompanied by spinorbit interaction. This interaction is weak and that is why the spin flips are rare events and the phase relaxation length for the spin part of the electron wave function is very long. But now, if the structure length $L$ is chosen to be $L_{\varphi}^{(s)}>L>L_{\varphi}^{(e)}$, it is possible to 
'wash out' the quantum interference related to phase coherence of the 'orbital part' of the wave function retaining at the same time the phase coherence of the spin part one and hence, to reveal the corresponding conductance oscillations of the microstructure. Such model was considered in the paper [4] where the simple theory of the quantum interference in a loop structure due to Larmor precession of electron spin was presented for the first time. The aim of this paper is to develop the approach further, discussing other aspects of the problem such as, for instance, electron scattering by the edges of the structure, the 'precession mechanisms' of spin relaxation which are characteristic ones for the crystal with lack of inversion symmetry, as well as their influence on the quantum interference of spins.

\section{Scattering by the edges of the structure}

We start with a generic microstructure with two end regions $(x<0$ and $x>L)$ and a middle region $0 \leq x \leq L$ consisting of two channels (Fig. 1),very similar to that one considered in [4]. The main difference however is the following. In the Ref [4], apart an external magnetic field $\mathbf{B}_{\mathbf{0}}$ in the plane of the microstructure, on the upper surface of one of the channels there was a regular periodic array of micromagnets which created and additional magnetic field. As it was mentioned in [4], the periodic magnetic field was not obligatory and that choice was motivated by the current interest in the study of electron motion in inhomogeneous magnetic fields on the nanometer scale. Here instead, even more simpler case of uniform magnetic field is considered, since the only thing which is really needed is that the magnetic fields to be different in the two arms of the loop. An additional advantage of the uniform magnetic field is that it is more simple and appropriate from the experimentalist's point of view.

Consider an electron enters the domain occupied by the magnetic field, say, from the left-end region. The electron's spin wave function is a coherent superposition of the spin-up and spin-down eigenstates, which are split in the magnetic field by the Zeeman energy $\Delta \varepsilon=g \mu_{B} B$, ( $g$ is the Lande factor). Coherent evolution under the spin Hamiltonian results in oscillations between these two eigenstates; classically this oscillation corresponds to precession of the spin vector at the Larmor frequency $\Delta \varepsilon / \hbar$. In other words, we consider the non-relativistic electron motion in the magnetic field as the motion of a classical top which precesses about the magnetic field. Since the magnetic fields are different in the two arms of the structure, the phase shifts acquired by the spin wave functions are also different and if one of the field (say, $B_{0}$ ) alters, it should lead to the specific conductance oscillations dependent on $B$.

So, the magnetic field $\mathbf{B}$ which affects the electron in the first arm of the structure is equal to $\mathbf{B}=\mathbf{B}_{\mathbf{0}}$, while in the other one it is equal to $\mathbf{B}=\mathbf{B}_{\mathbf{0}}+\mathbf{B}_{\mathbf{1}}$. Suppose the Hamiltonian of an electron is $H=H_{0}+H_{1}$, where

$$
H_{0}=1 / 2 m^{*}(\mathbf{p}-(e / c) \mathbf{A})^{2}+U(\mathbf{r}), H_{1}=-\mu_{B} \hat{\sigma} \mathbf{B},
$$

where $m^{*}$ is the electron effective mass, $\mathbf{A}$ is vector potential corresponding to the 
magnetic field $\mathbf{B}, \mu_{B}$ and $\hat{\sigma}$ are Bohr magneton and the spin operator respectively. We also assume that $U(\mathbf{r})$ describes conduction bands bending due to space charge and discontinuities of any band. Since $H_{0}$ does not depend on spin, the wave function is the direct product: $\Psi(\mathbf{r}, s)=\varphi(\mathbf{r}) \otimes \chi(s)$. Ever since for convenience we shall refer to $\varphi(\mathbf{r})$ as the 'orbital part' of the total wave function, keeping in mind that it corresponds to $H_{0}$ describing the charge-field interaction, and we shall refer to $\chi(s)$ as the spin-part of the wave function related to $H_{1}$, the spin part of the Hamiltonian $H$ in (1).

Let us now introduce the phase-relaxation length $L_{\varphi}^{(s)}$ for the spin part of the wave function, in just the same way as the one usually introduced for the 'orbital part', $L_{\varphi}^{(e)}$. As it was argued in the Introduction, the phase relaxation length $L_{\varphi}^{(s)}$ is much greater than $L_{\varphi}^{(e)}$. The proof of the statement can be found in Ref.[4], here we would like only to emphasize, that in accordance with our very cautions estimates, the phase relaxation time for the spin part of the wave function, $t \sim \tau_{\varphi}^{(s)}$ is about $\sim 2.2 \times 10^{-10} s$. This time is indeed much longer than the phase relaxation time for the orbital part one [7], and hence, if one takes into account only single scattering mechanism (scattering by phonons; this mechanism is known in the literature as Elliot-Yafet process [8]), one can safely assume $L_{\varphi}^{(s)}>>L_{\varphi}^{(e)}$ and consequently, that the structure length $L$ can be chosen to be $L_{\varphi}^{(s)}>L>L_{\varphi}^{(e)}$.

However, there is another mechanism which also, in principle, can contribute to the spin relaxation. This mechanism is the scattering by the surface and the edges of the structure.

Now our aim is to calculate the probability of the spin-flips caused by the electron scattering by the surface of the structure, since such spin-flips results in the 'washing out' of 'phase memory' of the spin-part of the electron wave function.

It is commonly used to treat the interaction of electrons with the surface of the sample in terms of a phenomenological parameter $\epsilon_{0}$ introduced for the first time by F. Dyson [9]. This quantity can be defined as the mean probability of spin-flip of the conduction electrons having the energy $\mathcal{E}=\mathcal{E}_{F}$ at their collision with the surface of the sample, averaged over the incident angles. Following [9], define $\epsilon_{0}$ as

$$
\epsilon_{0}=\hbar^{-1} \int d \Omega(\mathbf{J S})^{-1} \int \mathrm{d} \mathbf{k}^{\prime} \rho\left(\mathbf{k}^{\prime}\right) \delta\left[\mathcal{E}(\mathbf{k}, \sigma)-\mathcal{E}\left(\mathbf{k}^{\prime}, \sigma^{\prime}\right)\right]\left|\mathrm{V}_{\mathbf{k}^{\prime}, \sigma^{\prime} ; \mathbf{k}, \sigma}\right|^{2},
$$

where $\Omega$ is the incident solid angle, $\sigma, \sigma^{\prime}$ are the spin variables corresponding to the states before and after the collision with the surface, $\mathbf{k}, \mathbf{k}^{\prime}$ are the electron wave vectors before and after collision; $\mathbf{J}$ is the flux density of electrons incident on the surface from within; $\mathrm{S}$ is the surface area, $\rho\left(\mathbf{k}^{\prime}\right)$ is the density of states in the $\mathbf{k}$-space over the single spin; $V_{\mathbf{k}^{\prime}, \sigma^{\prime} ; \mathbf{k}, \sigma}$ is the matrix element of the perturbation operator responsible for the spin-flips; $\mathcal{E}(\mathbf{k}, \sigma), \mathcal{E}\left(\mathbf{k}^{\prime}, \sigma^{\prime}\right)$ are the electron energies in the corresponding quantum states.

It is quite obvious that the crystal potential which is supposed to be periodic deep inside the sample, changes abruptly at the edges and vicinity of the surface where it is not periodic altogether. These abrupt changes of the potential lead to the emerging of an electric field in a thin layer near the surface of the sample. The thickness of the layer 
can be estimated as to be 2-4 monolayers. Hence, if the electrons are moving in this layer, one can treat it as the quasi-two dimensional electron gas (2DEG).

Introduce now the space variables $x, y, z$ where $z$-axis is normal to the 2DEG. Suppose the external magnetic field $\mathbf{B}$ is directed along $y$-axis, $\mathbf{B}=(0, \mathrm{~B}, 0)$ and let the vector potential $\mathbf{A}$ to be: $\mathbf{A}=(\mathrm{Bz}, 0,0)$. Now the Hamiltonian of the electron moving in our 2DEG is of the form:

$$
H=\frac{1}{2 m^{*}}\left[\left(p_{x}-\frac{e B z}{c}\right)^{2}+p_{y}^{2}+p_{z}^{2}\right]+U(z)+\mu_{B} \hat{\sigma} B_{0} .
$$

Here $U(z)$ is the potential responsible for the space quantization in 2DEG in $z$-direction. The electron functions corresponding to such Hamiltonian can be written down as

$$
\Psi_{\mathbf{k} \sigma}(\mathbf{r})=\mathrm{C} \exp \left(\mathrm{ik}_{\mathrm{x}}+\mathrm{ik}_{\mathrm{y}}\right) \varphi\left(\mathrm{k}_{\mathrm{x}}, \mathrm{z}\right) \exp \left(-\mathrm{i} \mathcal{E}_{\mathrm{k}} / \hbar\right) \otimes \chi_{\sigma}
$$

and the normalizing conditions defined as

$$
\begin{gathered}
\int d x \psi_{k_{x}^{\prime} k_{y}^{\prime} \sigma^{\prime}}^{*}(x, y) \psi_{k_{x} k_{y} \sigma}(x, y)=\delta\left(\sigma, \sigma^{\prime}\right) \delta\left(\mathbf{k}-\mathbf{k}^{\prime}\right) \\
C=\left(\left(L_{x} L_{y}\right) \int_{-\infty}^{\infty}\left|\varphi\left(k_{x}, z\right)\right|^{2} d z\right)^{-1 / 2}
\end{gathered}
$$

Here $L_{x}, L_{y}$ are the structure sizes in $x, y$-directions.

As it was mentioned above, due to the potential $U(z)$, the strong electric field emerges at the surface of the structure . Obviously, the field is equal to $E=-e^{-1} \frac{\partial}{\partial z} U(z)$. If we suppose the absence of magnetic impurities in semiconductor, the only mechanism responsible for the spin-flips is the spin-orbit interaction. In the refrence frame of moving electron, the electric field generates an effective magnetic field of the form $\sim[\mathbf{E} \times$ $\left.(\mathrm{d} \mathbf{r} / \mathrm{dt}) \mathrm{c}^{-1}\right]$ which causes spin flips. The operator of spin-orbit interaction has the form (see Ref. [10]):

$$
V^{s o}=\frac{\hbar^{2}}{4 m^{* 2} c^{2}} \frac{\partial}{\partial z} U(z)\left(\sigma_{y} \frac{1}{i} \frac{\partial}{\partial x}-\sigma_{x} \frac{1}{i} \frac{\partial}{\partial y}\right),
$$

where $\sigma_{y}, \sigma_{x}$ are the corresponding Pauli matrices and this is the perturbation mentioned above.

After some manipulations carried out much in the same way as in Ref.[11], the dimensionless probability of the spin flips caused by the potential (4) and averaged over the incident angles, can be written as:

$$
\epsilon_{0}=\frac{1}{60}\left(m c^{2}\right)^{-2}\left(\cos ^{2}(\widehat{\vec{B}, \vec{n}})+1\right) I
$$

where $(\widehat{\vec{B}, \vec{n}})$ is the angle between $\mathbf{B}$ and the unit vector $\vec{n}$ normal to the surface and

$$
I=\left|\int_{-\infty}^{\infty} d z \varphi^{*}\left(-k_{x}, z\right) \frac{\partial U}{\partial z} \varphi\left(k_{x}, z\right)\right|^{2}
$$


Assuming, as it was done in [11], that the parameter $b=g \mu_{B} B / \mathcal{E}_{F} \ll 1$ ( $g$ is the Lande factor), it is possible to calculate integral in (5) even without having known the wave functions $\varphi_{k_{x} z}$ and the form of the potential $U(z)$. The authors of [11] considered the electron scattering by the surface in a metal where the Fermi energy is high enough and the parameter $b$ is small. However, in our case this assumption is no longer valid, since in 2DEG Fermi energy is not so high. So, we calculate the integral in (5) using the simple model of 'triangular potential well'. To this end, we write down the Schrödinger equation for the 'orbital part' of the wave functions (3) (it corresponds to the Hamiltonian (2) without Pauli's term) in the form:

$$
-\frac{\hbar^{2}}{2 m^{*}} \frac{\partial^{2} \varphi\left(k_{x}, z\right)}{\partial z^{2}}+\left[\frac{\hbar^{2} k_{x}^{2}}{2 m^{*}}+\frac{\hbar e B k_{x} z}{m^{*} c}+\frac{(e B z)^{2}}{2 m^{*} c^{2}}+U(z)-\mathcal{E}\left(k_{x}, k_{y}\right)\right] \varphi\left(k_{x}, z\right)=0,
$$

where $\mathcal{E}\left(k_{x}, k_{y}\right)=\mathcal{E}_{k_{x}}-\hbar^{2} k_{y}^{2} / 2 m^{*}$.

Now suppose the potential $U(z)$ has the form of triangular potential well, that is

$$
U(z)=\left\{\begin{array}{cc}
\infty, & z<0 \\
e E z, & z \geq 0
\end{array}\right.
$$

Suppose also so called quantum limit to occur, when only the lowest bands are occupied by the electrons. In the absence of a magnetic field, electrons in lower subbands (small subband index $n$ ) have a large mean free path, because the amplitude near the boundary is smaller and the scattering is less frequent. Therefore, the current is carried mostly by electrons in lower subband. In a magnetic field, electrons are pushed toward the boundaries by a Lorentz force and the scattering is enhanced. From now on we assume also the condition $\left(d_{0} / l_{B}\right)^{4} \ll 1$ is fulfilled. In the last relation $d_{0}=\left(3 \hbar^{2} \pi^{2} / 16 m^{*} e E\right)^{1 / 3}$ is the thickness of the 2DEG layer and $l_{B}=(\hbar c / e B)^{1 / 2}$ is the so called magnetic length. Then, the solution of the eigenvalue problem for Eq.(6) in the quantum limit approximation is of the form (see [12]):

$$
\begin{gathered}
\varphi\left(k_{x}, z\right)= \begin{cases}A i\left(\left[\frac{2 m^{*} e E}{\hbar^{2}}+\frac{2 e B k_{x}}{\hbar c}\right]^{1 / 3}\left[z-\frac{\mathcal{E}\left(k_{x}, k_{y}\right)-\hbar^{2} k_{x}^{2} / 2 m^{*}}{e E+\hbar e B k_{x} / m^{*} c}\right]\right), & z \geq 0 \\
0, & z<0\end{cases} \\
\mathcal{E}\left(k_{x}, k_{y}\right)-\hbar^{2} k_{x}^{2} / 2 m^{*}=\left(\hbar^{2} / 2 m^{*}\right)^{1 / 3}\left[\frac{9 \pi}{8}\left(e E+\frac{\hbar e B k_{x}}{m^{*} c}\right)\right]^{2 / 3},
\end{gathered}
$$

where $A i(\ldots)$ means Airy function (see, for instance, [13]). By means of the last formulae, we evaluated numerically the integral in (5) (see Fig. 2); at the numerical estimates of the spin flip probability we used also the next values of the parameters: $B \sim 0.1 T, k_{x} \sim k_{F}=0.3 \times 10^{7} \mathrm{~cm}^{-1}, E \sim 10^{3} \mathrm{Vcm}^{-1}, \mathrm{~m}^{*} \sim 0.1 \mathrm{~m}_{e}$. According to our estimates, the dimensionless probability of spin flip is equal approximately $\sim 3.7 \times 10^{-17}$. This result can be interpreted as follows. If we assume the number of spins $N_{0} \sim\left[\epsilon_{0}^{-1}\right]$, where $[x]$ stands for entier $(x)$, one spin among these $N_{0}$ undergoes the spin flip with probability closed to unit due to interaction considered above. If the number of spins 
is $N<N_{0}$, the probability to find one of them flip-flopped, is equal to $N / N_{0}$. The only relevant value of the dimension of time, by means of which this process can be characterized, is the time spent by electron within the domain where the interaction which causes spin flips, is essential. According to our estimates, the width of this domain $\left(l_{0}\right)$ is of the order of $l_{0}=0.33 \times 10^{-5} \mathrm{~cm}$, and the time which takes the electron to cross this region is about $t_{0} \sim l_{0} / v_{F}$. Let us assume the concentration of electron gas is equal to $\sim 10^{17} \mathrm{~cm}^{-3}$, the cross section of the channel of the structure in Fig.1 to be $1.0 \times 10^{-5} \times 1.0 \times 10^{-5} \mathrm{~cm}$ and the length of the channel to be $100 \mu \mathrm{m}$. As a result, the time of spin flip due to interaction with the surface can be estimated as $t_{0}\left(N_{0} / N\right) \sim 0.11 \mathrm{sec}$. This time, in accordance with the ideology of [4] can be identified with the spin phase relaxation time due to scattering by the surface. Now it is clear that the time of the spin flips due to scattering by the edges and the surface of the structure is extremely long. Considering two mechanisms of spin flips (the scattering by phonons and by surface or the edges) as independent, we can calculate the total probability of spin flip as the sum of the probabilities of these two events. As a result, the inverse spin relaxation time is the sum of the inverse relaxation times for two scattering mechanisms: $\left(\tau_{\varphi}^{(s)}\right)^{-1}=\left(\tau_{\varphi, p h}^{(s)}\right)^{-1}+\left(\tau_{\varphi, s}^{(s)}\right)^{-1}$, where $\tau_{\varphi, p h}^{(s)}, \tau_{\varphi, s}^{(s)}$ stand for the phase relaxation times due to scattering by the phonons and by surface, respectively. Since $\tau_{\varphi, p h}^{(s)} \ll \tau_{\varphi, s}^{(s)}$, the total spin relaxation time is practically equal to $\tau_{\varphi, p h}^{(s)}$. We can conclude that the scattering by the surface (edges) of the structure does not contribute essentially to the spin relaxation time and can be neglected. Therefore, $L_{\varphi}^{(s)}$ is indeed much greater than $L_{\varphi}^{(e)}$ and hence, the structure length can be chosen to be $L_{\varphi}^{(s)}>L>L_{\varphi}^{(e)}$.

\section{Precession mechanisms of spin phase relaxation}

So far our assertion, $L_{\varphi}^{(s)}>>L_{\varphi}^{(e)}$ was proved to be valid for two possible mechanisms of spin phase relaxation: Elliot-Yafet process and the spin relaxation due to surface scattering. However, there are another possible causes for the destruction of 'phase memory' of the spin part of electron wave function. These are, so called 'precession mechanisms' of spin-relaxation, for instance, D'yakonov-Perel mechanism [14]. This mechanism is characteristic one for the crystals of zinc-blende structure whose point group has no inversion symmetry: in a material with bulk inversion asymmetry (BIA) the electron energy bands are spin split for a given direction of the wave vector $\mathbf{k}$. There is also another very similar mechanism which is characteristic for the heterostructures and 2DEG layers, where the spin splitting may occur as a result of the structure inversion asymmetry (SIA) and which was first pointed out by Bychkov and Rashba [15].

Let us start with the D'yakonov-Perel mechanism. As is known, the diamond-type lattice consists of two similar face-centered cubic sublattices mutually penetrating each other. The zinc-blende type crystal lattices $\left(A^{I I I} B^{V}\right.$-type, for example) differ from the diamond-type one in that respect, that two sublattices are not identical: the first one 
consists of the $A$-type atoms, while another sublattice is of the $B$-type atoms. As a result, the point group of the zinc-blende structure does not involve the inversion and, as a consequence, the periodic part of the Bloch function satisfies no longer the condition $U_{-\mathbf{k}}(\mathbf{r})=\mathrm{U}_{\mathbf{k}}(-\mathbf{r})$. Hence, a twofold degeneracy is lifted and the electron energy bands are spin split in these materials for a given direction of the wave vector $\mathbf{k}$, even if the external magnetic field is not present. As a result, another mechanism of spin relaxation which was proposed for the first time in [14], becomes possible. We call this mechanism 'precession' mechanism of spin relaxation.

The spin splitting in $\mathbf{k}$-point is equal [14,16]:

$$
\Delta \varepsilon_{\mathbf{k}}=\eta\left(\mathbf{n}_{0}\right) \mathrm{k}^{3}, \quad \mathbf{n}_{0}=\mathbf{k} / \mathrm{k}
$$

where $\eta\left(\mathbf{n}_{0}\right)=\eta_{0}|\kappa(\mathbf{n})|, \quad \kappa_{\mathrm{x}}=\mathrm{n}_{0 \mathrm{x}}\left(\mathrm{n}_{0 \mathrm{y}}^{2}-\mathrm{n}_{0 \mathrm{z}}^{2}\right)$ and the other components of $\kappa$ can be obtained by means of cyclic permutation of indices.

The spin relaxation time could be estimated as follows. Let the initial electron state characterised by $\mathbf{k}$ be polarized along some axis, say $\mathbf{a}$, which does not coinside with $\kappa(\mathbf{n})$ and let its spin state be $\sigma=+1 / 2$. Regarding the spin splitting $\Delta \varepsilon_{\mathbf{k}}$, this state is not longer the eigenstate and as time passes by, it changes. It means, as it was already mentioned above, that another component with $\sigma=-1 / 2$ mixes up to the state with $\sigma=+1 / 2$. The coherent mixture of these two states corresponds, in the classical picture, to the spin precession about the $\kappa(\mathbf{n})$-axis with the frequency $\Omega_{\mathbf{k}}=\Delta \varepsilon_{\mathbf{k}} / \hbar$.

The spin relaxation arises due to the momentum relaxation which is always present to some extent and accompanies the spin precession. Since $\Omega_{\mathbf{k}} \tau_{\mathbf{p}}<<1$, during the elapse of time between two successive scattering events the electron spin revolves only by a small angle $\sim \Omega_{\mathbf{k}} \tau_{\mathbf{p}}$. At each scattering wave vector $\mathbf{k}$ changes randomly and as a result, $\kappa(\mathbf{n})$ changes too. It means, that the direction of spin precession axis changes randomly also, as the electron moves through the crystal (see Fig.3). After many scattering events the initial 'spot'corresponding to the initial direction of precession axis spreads over unit sphere $S^{2}$; so, one can treat this process as some kind of the diffusion over $S^{2}$ with the angular diffusion coefficient $\mathcal{D}_{\mathrm{s}}$ of about $\sim\left(\Omega_{\mathbf{k}} \tau_{\mathbf{p}}\right)^{2} / \tau_{\mathbf{p}} \cdot[16]$ The time $T$ which is needed for the initial 'spot' to run over $S^{2}$ uniformly, can be estimated as $\left(\mathcal{D}_{\mathrm{s}} T\right)^{1 / 2} \sim 4 \pi$, and hence,

$$
T \sim(4 \pi)^{2} / \Omega_{\mathbf{k}}^{2} \tau_{\mathbf{p}}
$$

Intuitively it is clear that $T>\tau_{\mathbf{p}}$, since one need to have many scattering events, in order the initial 'spot' to run over entire $S^{2}$.

More precise calculations (see [8]) give the next formula for $\Omega_{\mathbf{k}}$ :

$$
\Omega_{k}^{2}(\varepsilon)=\frac{16}{315}\left(\frac{\Delta}{\hbar}\right)\left(\frac{A^{\prime}}{\hbar^{2} / 2 m_{e}}\right)\left(1-\frac{m^{*}}{m_{e}}\right) \times \frac{1}{(1+\lambda)\left(1+\frac{2}{3} \lambda\right)}\left(\frac{m^{*}}{m_{e}}\right)\left(\frac{\varepsilon}{E_{g}}\right)^{3},
$$

where $\lambda=\Delta / E_{g}, \Delta$ is the spin-orbit splitting, $E_{g}$-energy gap and the parametr $A^{\prime}$ is defined as follows

$$
A^{\prime}=2 \frac{\hbar^{2}}{m_{e}^{2}} \sum_{u} \frac{<s\left|P_{x}\right| u><u\left|P_{y}\right| z>}{E_{c}-E_{u}} .
$$


In the last formula $s$ is a function transformed in accordance with $\Gamma_{1}$ irreducible representation of zinc-blende structure space group, $u$ is the periodic part of the Bloch function and $z$ is the function which transforms under the tetrahedral group transformations just like $P_{z}$ atomic functions .

According to G.Fishman and G.Lampel [8], $\Omega_{k}^{2}(\varepsilon)$ for $G a A s$ is approximately equal to $10^{18} \varepsilon^{3}$, if $\Omega$ is in rad per second and $\varepsilon$ is in $e V$.

Since we deal with mesoscopic loop structure and 2DEG, it is also important to take into account another very similar mechanism of spin-splitting occurring in 2D electron gas and which was pointed out by Yu.Bychkov and E.Rashba [15]. They noticed, that in heterostructures and surface layers, there is lack of inversion asymmetry due to the existence of interfaces. This type of asymmetry could be called 'structure inversion asymmetry'(SIA). The corresponding spin-orbit Hamiltonian, according to [15] is of the form:

$$
H_{S O}=\xi[\boldsymbol{\sigma} \times \mathbf{k}] \cdot \boldsymbol{\nu}
$$

where $\boldsymbol{\sigma}$ are the Pauli matrices, $\boldsymbol{\nu}$ is a unit vector perpendicular to the surface and $\xi$-some constant whose numerical value can be established by the cyclotron resonance data.

As in previous case, the operator $H_{S O}$ lifts the twofold spin degeneracy at $\mathbf{k} \neq 0$ and determines the spin-orbit band splitting near $\mathbf{k}=0$

Just in the same way as previously, this mechanism leads to the precession of spin axis and because of $\mathbf{k}$-dependence and electron scattering, to the diffusion of the initial 'spot' corresponding to the initial state of spin precession axis over entire $S^{2}$-surface. The frequency of the precession is equal to $\Omega_{\mathbf{k} \nu}=\Delta \varepsilon_{\mathbf{k} \nu} / \hbar$, where $\Delta \varepsilon_{\mathbf{k} \nu}$ corresponds to the spin splitting due to $H_{S O}$ (we add here subscript $\boldsymbol{\nu}$ in order to distinguish this mechanism of spin precession from the first one).

It is clear, that if the semiconductor, of which the structure in question is made, is of zinc-blende type, we could expect these two mechanisms doing simultaneously together. What is less obvious and which was first pointed out by P.Pfeffer and W.Zawadzki [17], that there is no simple additivity of these two mechanisms: $\Delta \varepsilon_{\text {tot }} \neq \Delta \varepsilon_{\mathbf{k}}+\Delta \varepsilon_{\mathbf{k} \nu}$ (subscript 'tot' stands for 'total"). According to [17], the $\Delta \varepsilon_{\text {tot }}$ depends on the subtleties of semiconductor band structure and electron density in 2DEG. Now proceed to the estimates of the spin relaxation time for the materials of zinc-blende type, such as $G a A s, I n S b$ and InAs. To the authors knowledge, the most reliable data are known for the first of these three materials, so let us start from the estimates for GaAs. If one starts with D'yakonov-Perel mechanism which corresponds to BIA, then one can easily estimate $\Omega_{\mathbf{k}}(\mathrm{GaAs})$ as to be equal $\sim 3.088 \times 10^{11} \mathrm{~Hz}$. Taking into account SIA and its generalization proposed by Pfeffer and Zawadzki, supposing the electron density in 2DEG to be equal $N_{s}=10^{12} \mathrm{~cm}^{-2}$, one get from the data of [17] that $\Delta \varepsilon_{\text {tot }}(\mathrm{GaAs})$ is about $0.46 \mathrm{meV}$. Introducing $\Omega(\mathbf{k}, \boldsymbol{\nu})=\Delta \varepsilon_{\text {tot }} / \hbar$ and using the approach discussed above (see formula (7)) we can evaluate the spin relaxation time in the framework of the generalized model which includes both mechanisms. The time, according to our estimations, is approximately equal $T \sim 3.2 \times 10^{-8}$. 
Using the data of [8], one can get for the ratios $\Omega_{\mathbf{k}}(G a A s) / \Omega_{\mathbf{k}}(\operatorname{InSb})$ and $\Omega_{\mathbf{k}}(G a A s) /$ $\Omega_{\mathbf{k}}(\operatorname{In} A s)$ the values: 0.378 and 1.58, respectively. As a consequence, $\Omega_{\mathbf{k}}(\operatorname{In} S b) \sim$ $8.037 \times 10^{11} \mathrm{~Hz}$ and $\Omega_{\mathbf{k}}($ InAs $) \sim 1.216 \times 10^{11} \mathrm{~Hz}$. Unfortunately, nothing is known for certain about SIA for these materials, as well as for its Pfeffer-Zawadzki generalization. Let us suppose, however, that the last one leads to the same consequences for these two materials as in previous case of gallium arsenide. Then, at $N_{s}=10^{12} \mathrm{~cm}^{-2} \Delta \varepsilon_{\text {tot }}$ should be about twice as great as $\Delta \varepsilon_{\mathbf{k}}$, and the same is valid for $\Omega(\mathbf{k}, \boldsymbol{\nu})$. As a result, we have for $T(\operatorname{InSb}) \sim 2.4 \times 10^{-8} s$ and for $T(\operatorname{In} A s) \sim 1.0 \times 10^{-6} s$. Of course, the last results are only very rough estimates.

The natural question which is to be answered now is this: how does this spin relaxation time relate to the spin phase relaxation time $\tau_{\varphi}^{(s)}$ introduced above? One can relate the spin relaxation time $T$ to the phase relaxation time simply in the following way. Since our structure is in external magnetic field, the frequency of spin precession is the sum of $\Omega(\mathbf{k}, \boldsymbol{\nu})$ and the Zeeman frequency $\Omega_{Z}=g \mu_{B} B / \hbar: \Omega=\Omega(\mathbf{k}, \boldsymbol{\nu})+\Omega_{Z}$, where $g$-is Lande factor. This frequency is a bit different for different electrons, since $\Omega(\mathbf{k}, \boldsymbol{\nu})$ depends on the electron's quasi-momentum k. Thus, the electron scattering means that the 'oscillator' characterized by $\Omega$ is 'triggering' all the time from the frequency $\Omega^{\prime}\left(\mathbf{k}^{\prime} \boldsymbol{\nu}\right)+\Omega_{Z}$ to another one, $\Omega^{\prime \prime}\left(\mathbf{k}^{\prime \prime} \boldsymbol{\nu}\right)+\Omega_{Z}$, than to $\Omega^{\prime \prime \prime}\left(\mathbf{k}^{\prime \prime \prime} \boldsymbol{\nu}\right)+\Omega_{Z}$ and so on. Since each of these frequencies differ from one another only by some small value, each single scattering event leads only to small 'phase aberration'. Thus, the single 'triggering'does not yet introduce the irreversibility and phase destruction. However, after many scattering events not only the direction of initial quasi-momentum changes, but its absolute value changes too. The last one introduces the necessary element of irreversibility and means the destruction of 'phase memory' of the spin part of electron wave function. The complete phase destruction occurs after the elapse of time $\tau_{\varphi, P Z}^{(s)} \sim T \simeq\left(\Omega_{t o t}^{2} \tau_{\mathbf{p}}\right)^{-1}$ (we add here the subscript 'PZ', to emphasize that we used the generalized Pfeffer-Zawadzki model).

Now compare the Elliot-Yafet (EY) and precession mechanisms and estimate their combined action on the spin phase relaxation. Note also that this kind of spin phase relaxation can be termed as environmental decoherence [1].

To this end let us make at first some comments concerning calculations of the spin phase relaxation time which is due to EY-mechanism. In the paper [4] the next formula for $\tau_{\varphi, p h}^{(s)}$ was derived:

$$
\tau_{\varphi, p h}^{(s)} \sim\left(\hbar^{2} / \tau \varepsilon_{\text {int }}^{2}\right) \ln 2 \times \tanh (\beta \varepsilon / 2),
$$

where $\beta=\left(k_{B} T\right)^{-1}, \varepsilon$ - is the Zeeman splitting, $\tau$ - has the meaning of 'electron-phonon collision time' and $\varepsilon_{\text {int }}$ is an 'interaction energy' which characterizes an interaction between two-level quantum sub-system and the phonon bath. In the paper [4] only the lower boundary for $\tau_{\varphi, p h}^{(s)}$ was established, which nevertheless turned out to be much greater than $\tau_{\varphi}^{(e)}$ (see also the final passage of Sec.2). It is possible to get more rigorous theory which could make more precise calculations possible. The theory could, for instance, utilize the spin-orbit operator of the form similar to (4), in which however, 
$\nabla U$ - term should be substituted by $\nabla V_{\mathbf{q}}(\mathbf{r})$, where $V_{\mathbf{q}}(\mathbf{r})$ stands for the perturbation caused by a single phonon. Such theory is however beyond the scope of the paper, the more so that in this eventual theory there would be some other parameters which in their turn are not absolutely precise. Since our aim is only to show that $\tau_{\varphi, p h}^{(s)}>>\tau_{\varphi}^{(e)}$, we restrict our treatment rather to estimates. But in order to take into account the difference between $G a A s, \operatorname{In} S b$ and $\operatorname{In} A s$, we include into $\varepsilon_{\text {int }}$ the factor $(\bar{Z} \alpha)$, where $\bar{Z}$ is the mean atomic number of the corresponding compound and $\alpha=e^{2} / \hbar c$. This factor is important, because the role of spin-orbit interaction increases as the atomic number $Z$ increases [16].

The results of our calculations are summed up in Fig. (4) and in Table 1. In Fig. (4) the spin phase relaxation times which are due to EY mechanism are shown for GaAs, InSb and InAs as the function of external magnetic field. In Table 1 the spin phase relaxation times, due to EY mechanism and for two chosen values of magnetic field are compared to the relaxation times due to precession mechanism. Obviously, one can treat these mechanisms as independent and hence,

$$
\frac{1}{\tau_{\varphi}^{(s)}}=\frac{1}{\tau_{\varphi, p h}^{(s)}}+\frac{1}{\tau_{\varphi, P Z}^{(s)}}
$$

Now it is clear that at least in accordance with our calculations, for GaAs the EYmechanism dominates for magnetic fields $B \leq 1 T$, because $\tau_{\varphi, p h}^{(s)}$ is the shortest time. For InSb one can conlude from the data of Table 1, that for small magnetic field precession mechanism dominates, because $\tau_{\varphi, P Z}^{(s)}$ is the shortest time, while for $B \sim 1 T$ EY-mechanism becomes dominating.

Table 1

\begin{tabular}{|c|c|c|c|}
\hline \multirow{2}{*}{ semiconductor } & \multicolumn{2}{|c|}{$\tau_{\varphi, p h}^{(s)}, s$} & \multirow{2}{*}{$\tau_{\varphi, P Z}^{(s)}, s$} \\
\cline { 2 - 3 } & $B=0.1 T$ & $B=1 T$ & \\
\hline GaAs & $2.6 \times 10^{-9}$ & $2.3 \times 10^{-8}$ & $3.2 \times 10^{-8}$ \\
\hline InSb & $4.27 \times 10^{-8}$ & $1.3 \times 10^{-7}$ & $2.4 \times 10^{-8}$ \\
\hline InAs & $2.9 \times 10^{-9}$ & $2.21 \times 10^{-7}$ & $1.0 \times 10^{-6}$ \\
\hline
\end{tabular}

It is interesting to compare our results with the experimental ones. According to the recent data obtained by D.Awschalom, J.Kikkawa and others [1,2], the spin decay due to environmental decoherence can exceed even $100 \mathrm{~ns}$ and it is in excellent agreement with our calculations. So, again we can conclude that the condition $L_{\varphi}^{(s)}>L>L_{\varphi}^{(e)}$ can be satisfied.

\section{Calculation of the transmission coefficient}

The current $I$ through the structure considered in the Section 2, for the small applied potential $V$, can be written as $[4,18]$ :

$$
I=\frac{2 e}{h} \int d \mathcal{E} \int\left(w_{z} d k_{z} / 2 \pi\right)\left[f(\mathcal{E})-f(\mathcal{E}+e V) \sum_{n^{\prime}, n^{\prime \prime}}\left|T_{n^{\prime}, n^{\prime \prime}}\right|^{2} .\right.
$$


Here $w_{z}$ is the width of the structure in the $z$-direction, $T_{n^{\prime}, n^{\prime \prime}}$ is the transmission coefficient from the state $n^{\prime}$ in the left-hand end to the state $n^{\prime \prime}$ in the right-hand end, $\mathcal{E}$ and $k_{z}$ are the energy and the transverse wave vector of the electrons as they enter from the left-hand end.

The approach to calculation of the transmission coefficient $T_{n^{\prime}, n^{\prime \prime}}$ was outlined in Ref.[4]; here for reader's convenience, we add only few comments.

Since the length $L$ of the structure is supposed to be greater than $L_{\varphi}^{(e)}$, the key idea is to devide the structure into sections of the length smaller than $L_{\varphi}^{(e)}$. Then one can combine these successive scatters, considering the transport through the states $k^{\prime}, k^{\prime \prime}$ as incoherent, while the transport through the states $\sigma^{\prime}, \sigma^{\prime \prime}$ as coherent because of $L<L_{\varphi}^{(s)}$. As a result, the expression for the transmission coefficient is of the form (see[4]):

$$
T=t^{\prime}\left[I-\operatorname{Pr} P^{\prime} r^{\prime}\right]^{-1} P t,
$$

where the subscripts $\sigma^{\prime}, \sigma^{\prime \prime}$ are dropped out. Here $I$ is the unit matrix, $t$ is $4 \times 1$ matrix describing the transmission from the left-hand end into the two channels, while $t^{\prime}$ is $1 \times 4$ matrix describing the transmission from the channels into the right-hand end. Similarly, $r$ and $r^{\prime}$ are $4 \times 4$ matrices describing the reflections at the two junctions of the channels back into the channels. Matrices $P$ and $P^{\prime}$ describe forward and reverse propagation of the electron wave through the channels 1 and 2 , respectively.

The general remark which should be made, concerns the validity of Landauer-Büttiker formalism in this particular case. It is known [19] that this formalism provides a rigorous framework for the description of mesoscopic transport as long as transport across the structure is coherent. For noncoherent transport, however, the situation is more complicated, if there is a 'vertical flow' of electrons, that is the electron transitions from one energy to another. In that sence our case is rather intermediate one: the transport through the states $\sigma^{\prime}, \sigma^{\prime \prime}$ is coherent, while through the states $k^{\prime}, k^{\prime \prime}$ is incoherent. Luckily, sometimes even if 'vertical flow' is pressent, it can be neglected (see[19,p.111]) provided transmission functions are approximately constant over the energy range where transport occurs:

$$
\left(\mu_{1}+\mu_{2}\right)+\left(n \times k_{B} T\right)<<\varepsilon_{c},
$$

where $1 \leq n \leq 5, \varepsilon_{c}$ is the correlation energy.

To estimate correlation energy, one can use simple relation: $\varepsilon_{c} \sim \hbar / \tau_{\varphi}^{(e)}$. Since $\tau_{\varphi}^{(s)}>>$ $\tau_{\varphi}^{(e)} \sim 10^{-12}-10^{-13}$, correlation energy is about 0.6-6 meV. So, we assume the voltage $V$ applied to the structure to be sufficiently small, in order to satisfy the condition above.

Since we assume the length of the structure $L>L_{\varphi}^{(s)}$, there are no spin flips in two channels considered and hence, among the 16 matrix elements of $r$-matrix (as well as $r^{\prime}$ ) 8 entries are equal to zero.

In order to construct $P$ and $P^{\prime}$, it is necessary to note that the spin parts of the wave functions acquire the phase factors due to Larmor spin precession about $\mathbf{B}$-axis. Since magnetic field in the channels are different, these phase factors are also different. 
One can treat the states 'spin up' and 'spin down' as the two opposite points on a unit sphere $\mathcal{S}^{2}$ which can be transformed one into another under rotation by an angle $\varphi= \pm \pi$ about some axis $\mathbf{a}$. Introduce also formally $\mathbf{b}$-axis which is a unit vector of the precession axis: $+\mathbf{b}$ corresponds to the electron propagation from $x=0$ to $x=L$ while $-\mathbf{b}$ corresponds to reverse propagation, and $\theta_{1}$ and $\theta_{2}$ are the phase acquired by spin part of the wave functions in the channels 1 and 2, respectively. Then the matrix elements describing the phase shifts in the two channels can be written as:

$$
\begin{gathered}
P_{ \pm 1}=\exp \left( \pm i \varphi_{a}\right) \exp \left(i \theta_{1, b}\right), P_{ \pm 1}^{\prime}=\exp \left( \pm i \varphi_{a}\right) \exp \left(-i \theta_{1, b}\right) \\
P_{ \pm 2}=\exp \left( \pm i \varphi_{a}\right) \exp \left(i \theta_{2, b}\right), P_{ \pm 2}^{\prime}=\exp \left( \pm i \varphi_{a}\right) \exp \left(-i \theta_{2, b}\right) .
\end{gathered}
$$

The idea of (8)-(9) is to express the elements of the matrices $P, P^{\prime}$ as the two rotations about two independent axis. Then, these objects are nothing else but the unitary quaternions [20]. As is known [20], any quaternion can be written in the form $q=c_{0}+i_{1} c_{1}+i_{2} c_{2}+i_{3} c_{3}=\sum_{\alpha=0}^{3} i_{\alpha} c_{\alpha}$, where $i_{0}=1$ and $i_{1}^{2}=i_{2}^{2}=i_{3}^{2}=i_{1} i_{2} i_{3}=-1$. However, it is possible also to define, for instance, $i_{1}, i_{2}$ as

$$
i_{1}=\left(\begin{array}{cc}
0 & 1 \\
-1 & 0
\end{array}\right), i_{2}=\left(\begin{array}{cc}
0 & i \\
i & 0
\end{array}\right)
$$

where $i$ is the ordinary complex square root of -1 , thus forcing

$$
i_{3}=i_{1} i_{2}=\left(\begin{array}{cc}
i & 0 \\
0 & -i
\end{array}\right) .
$$

If these three matrices are multiplied by $-i$, one obtains Pauli spin matrices. Thus, the quaternion $q$ could have been identified with the complex 2-by-2 matrix

$$
\left(\begin{array}{cc}
c_{0}+i c_{3} & c_{1}+i c_{2} \\
-c_{1}+i c_{2} & c_{0}-i c_{3}
\end{array}\right)=\left(\begin{array}{cc}
u & v \\
-v^{*} & u^{*}
\end{array}\right)
$$

where $u$ and $v$ are complex numbers with complex conjugates $u^{*}$ and $v^{*}$. Replacing 0,1 and $i$ in these complex matrices by

$$
\left(\begin{array}{ll}
0 & 0 \\
0 & 0
\end{array}\right),\left(\begin{array}{ll}
1 & 0 \\
0 & 1
\end{array}\right),\left(\begin{array}{cc}
0 & 1 \\
-1 & 0
\end{array}\right),
$$

respectively, one can obtain a representation of quaternions as 4-by-4 matrices.

Since two channels 1 and 2 are supposed to be isolated, in this way the matrices $P$ and $P^{\prime}$ can be represented as the diagonal $4 \times 4$-matrices with the diagonal elements defined by (8)-(9).

After a great deal of algebra (see Ref.[4] ), we have:

$$
\begin{array}{r}
|T|^{2}=\left|a_{1}\right|^{2}+\mid \\
\left|a_{2}\right|^{2}+\left|a_{3}\right|^{2}+\left|a_{4}\right|^{2}+\left(a_{1}^{*} a_{3}+a_{1} a_{3}^{*}+a_{2}^{*} a_{4}+a_{2} a_{4}^{*}\right)+\left(a_{1}^{*} a_{2}\right. \\
\left.+a_{1} a_{2}^{*}+a_{2}^{*} a_{3}+a_{2} a_{3}^{*}+a_{3}^{*} a_{4}+a_{3} a_{4}^{*}\right) \cos \Delta \theta, \Delta \theta=\theta_{1}-\theta_{2},
\end{array}
$$


where $a_{i},(i=1,2,3,4)$ do not depend on $\theta_{1}, \theta_{2}$ and are the complicated functions of $r_{i j}$, $r_{i j}^{\prime}, t_{i}, t_{i}^{\prime}$.

Note, that the cosine dependence of the transmission coefficient on phase difference in the last expression is the direct consequence of the quaternion representation of the propagation matrices, $P$ and $P^{\prime}$.

It is interesting to note that the same cosine dependence on phase defference was obtained in the experiments with two interfering neutron beams [21]. These results are now considered as the direct verification of the $4 \pi$-symmetry of spinors. Since Dirac's equation can be written in quaternion representation (see, for instance, [22]), we can conclude that our result is in total agreement with $4 \pi$-symmetry of spinors.

\section{Calculation of the phase shift}

Now let us suppose the mesoscopic structure under consideration is made od the zincblende type semiconductor and proceed to the calculation of the phase shift acquired by the spin part of the electron wave function.

Consider the non-relativistic motion of the particle (electron) with the spin $|s|=1 / 2$ in a two- component magnetic field: $\mathbf{B}=\mathbf{B}_{\mathbf{0}}+\mathbf{B}_{\mathbf{1}}, \mathbf{B}_{\mathbf{0}}=\left(0, \mathrm{~B}_{0}, 0\right)$, and $\mathbf{B}_{\mathbf{1}}=\left(0,0, \mathbf{B}_{1}\right)$, where $B_{1}$ is an additional uniform magnetic field in one of the channels of the structure. The spin part of electron wave function can be considered as a two-component vector defined by the pair of functions $\chi(\mid \uparrow>)$ and $\chi(\mid \downarrow>)$ which stand for the probability amplitudes of the two possible orientations of spin. The spin operator $\hat{\sigma}\left(\sigma_{x}, \sigma_{y}, \sigma_{z}\right)$ is defined in terms of Pauli matrices:

$$
\sigma_{x}=\left(\begin{array}{ll}
0 & 1 \\
1 & 0
\end{array}\right), \sigma_{y}=\left(\begin{array}{rr}
0 & -i \\
i & 0
\end{array}\right), \sigma_{z}=\left(\begin{array}{rr}
1 & 0 \\
0 & -1
\end{array}\right)
$$

Thus, we can treat the mean value of the magnetic moment of the electron moving within the channels of mesoscopic structure as the classical quantity $\mathbf{P}=\langle\sigma\rangle$, its evolution under magnetic field being defined by the equation:

$$
\frac{d \mathbf{P}}{d t}=\gamma^{*}[\mathbf{P}, \mathbf{B}]
$$

where $\gamma^{*}=e / m c$ is the electron gyromagnetic constant.

In other words, the vector $\mathbf{P}$ can be treated as classical magnetic top and, if this classical top having the initial orientation $\mathbf{P}_{\mathbf{0}}=\left(\mathrm{P}_{\mathrm{x}}^{0}, \mathrm{P}_{\mathrm{y}}^{0}, \mathrm{P}_{\mathrm{z}}^{0}\right)$ enters magnetic field $\mathbf{B}=$ $\left(\mathrm{B}_{\mathrm{x}}, \mathrm{B}_{\mathrm{y}}, \mathrm{B}_{\mathrm{z}}\right)$, it begins to precess about magnetic field with the frequency $\Omega=\gamma^{*} B$, where $B=\sqrt{B_{x}^{2}+B_{y}^{2}+B_{z}^{2}}$.

It is interesting to note that despite its purely quantum character, the spin of the particle during its movement in external fields often can be treated classically. The accuracy of such treatment can be estimated by means of Heisenberg uncertainty relation, since classical treatment is possible if one can neglect the commutator $[\mathbf{r}, \mathbf{p}]$ where $\mathbf{p}$ is 
the particle momentum operator. So, the measure of accuracy of the classical approximation is $|\Delta p| / p . \Delta p$ in our case can be estimated as $\sim m \Delta v=m\left(v^{2} / l_{B}\right) \Delta t$, where $l_{B}=\sqrt{\hbar c /|e| B}$ is the magnetic length and $\Delta t \sim 2 \pi / \omega_{c}, \omega_{c}=|e| B / m c$ is the cyclotron frequency, while $|\Delta p| / p \sim 2 \pi m v_{F} \sqrt{m c} / \sqrt{\hbar|e| B}$. Assuming $v_{F} \sim 3 \times 10^{7} \mathrm{cms}^{-1}$ and $B \sim 0.1 T$, we have $|\Delta p| / p \approx 1.26 \times 10^{-10}$. Therefore, indeed to a good approximation, we can treat the evolution of vector $\mathbf{P}$ as the evolution of the classical magnetic top under external magnetic field.

Let us introduce now the phase of precessing spin by means of the formula

$$
\theta(v, x)=\mu_{B} g / \hbar \int_{0}^{x} B(v, x) d t=\gamma^{*} / v \int_{0}^{x} B d x^{\prime} .
$$

Since magnetic fields, $\mathbf{B}_{\mathbf{0}}$ and $\mathbf{B}_{\mathbf{1}}$ are uniform, the calculation of the phase shift $\Delta \theta$ can easily be done.

Moreover, it is clear that under certain conditions including appropriate structure length $L$, electron velocity and the values of magnetic fields $B_{0}, B_{1}$, the phase shift $\Delta \theta=\theta_{2}-\theta_{1}$ can be multiple of $\pi$. Indeed,

$$
\Delta \theta=\theta_{2}-\theta_{1}=n \pi=(\gamma L / v)\left(\sqrt{B_{0}^{2}+B_{1}^{2}}-B_{0}\right), n=1,2 \ldots
$$

If the values of $B_{1}, L, v, n$ are given, the value of $B_{0}$ which is needed for the $\Delta \theta$ to be equal of multiple of $\pi$ can be easily calculated:

$$
B_{0}=\left|\frac{\gamma^{*} L}{2 n \pi v} B_{1}^{2}-\frac{n \pi v}{2 \gamma^{*} L}\right| .
$$

Hence, changing the external magnetic field $B_{0}$, one can change the phase shift and the quantum interference from constructive to destructive one and back. Also it is seen that $\Delta \theta=\theta_{2}-\theta_{1}=f\left(B_{0}, B_{1}, v\right)$ is the function of $B_{0}, B_{1}, v$. That is, the phase shift generally speaking is different for the electrons with different velocities. At first sight, this makes matters worse, because it means that the 'interference pattern' should be blurred. One should remember, however, that the temperature is considered to be sufficiently low. It means that most of the electrons carrying current are on the Fermi surface, that is the electron distribution function $f(\varepsilon)=\chi\left(\varepsilon_{F}-\varepsilon\right)$ and $v=v_{F}$, where $\chi(\ldots)$ is the Heaviside step-like function, $\varepsilon_{F}, v_{F}$ are the Fermi energy and Fermi velocity, respectively. Someone can be temptated to substitute $v$ in the last formula by drift velocity. But the condition $L_{\varphi}^{(s)}>L>L_{\varphi}^{(e)}$ does not imply that electrons undergo so many collisions that the drift velocity arises. Remember, that the concept of drift velocity is relevant to macroscopic samples, where electrons undergo a great many collisions under which (and an external electric field) the drift velocity can only be formed. Here instead, we have mesoscopic structure where electrons suffer only a few collisions after which the phase coherence of the orbital part of the electron wave functions in the two arms of the structure is destroyed. 
As a result, we can substitute in (13) $v$ by $v_{F}$ assuming that most of the electrons carrying current are on the Fermi surface and a few collisions which they undergo during their movement withing the channels of the structure do not change essentially their flight time which is still approximately equal to $\sim L / v_{F}$.

So, the calculation by means of (10) taking into account the expression for $|T|^{2}$, now can easily be done and we have:

$$
I=(2 e / h) K\left(A+D \cos \Delta \theta\left(v_{F}\right)\right)
$$

where $K, A, D$ are the coefficients dependent on the peculiarities of the structure. Now it is clear that changing $B_{0}$ one can approach very deep modulation of the conductance and since $A \sim D$, the 'contrast' of the 'interference pattern' is defined only by the ratio $\sqrt{\frac{\varepsilon_{F}-k_{B} T}{\varepsilon_{F}}}$.

So, we conclude that if the structure length $L$ is chosen to be $L_{\varphi}^{(s)}>L>L_{\varphi}^{(e)}$, it is indeed possible to 'wash out' the quantum interference related to phase coherence of the 'orbital part' of electron wave function, retaining at the same time that one related to the phase coherence of the spin part. Moreover, we can expect this 'interference pattern' and corresponding current (or conductance) modulation to be strong enough in order to be observed.

It is also interesting to note that current (and conductance) oscillations generally speaking, are not periodic with respect to $B_{0}$, the magnetic field by means of which these oscillations are controlled. This is due to the fact, that while the second term in (13) is linearly proportional to $n$, the first one is inversely proportional to it. Another interesting feature of (13) is the quadratic dependence of $B_{0}$ on the static field $B_{1}$. By means of this formula one can easily calculate $\Delta B_{0}$, the changing in magnetic field $B_{0}$ which is needed to change $\Delta \theta$,for example, from $\pi$ to $2 \pi$. The corresponding data for three zinc-blende type semiconductors, two chosen values of $B_{1}$ and $L=1.5 \times 10^{-2} \mathrm{~cm}$ are presented in Table 2.

Table 2

\begin{tabular}{|c|c|c|}
\hline \multirow{2}{*}{ semiconductor } & \multicolumn{2}{|c|}{$\Delta B_{0}, T$} \\
\cline { 2 - 3 } & $B_{1}=0.1, T$ & $B_{1}=0.5, T$ \\
\hline$G a A s$ & 0.002 & 0.91 \\
\hline$I n S b$ & 3.54 & 88.68 \\
\hline InAs & 1.07 & 26.75 \\
\hline
\end{tabular}

Keeping in mind the possibility of experimental verification of the theory presented in the paper, one can conclude from the Table 2, that some materials and some values of magnetic field $B_{1}$ are more sutable than the others. Perhaps GaAs is the best material for that purpose, while if $B_{1}>0.2 T$ such experiment for InSb and InAs becomes rather impossible. 


\section{Conclusion}

A simple theory of the quantum interference due to Larmor precession of an electron spin in a loop structure is presented in this paper. We investigate different mechanisms of environmental decoherence, such as edge scattering, Elliot-Yafet and precession mechanisms of spin relaxation, as well as their influence on the quantum spin interference in such structure. It turns out, that the time of spin phase relaxation due to edge scattering is very long and this mechanism can be neglected, while the other two are essential. The EY - and precession mechanisms thus determine the spin phase relaxation. As it is shown, even if EY - and precession mechanisms do together, it is still possible nevertheless, to satisfy the condition $L_{\varphi}^{(s)}>L>L_{\varphi}^{(e)}$. The last one determines the 'spin ballistic' transport in the structure in question, that is, the phase relaxation length $L_{\varphi}^{(s)}$ of the spin part of the electron wave function is assumed to be greater than the microstructure length. If in one of the microstructure's arms there is an additional magnetic field, the spin wave function acquires a phase shift due to additional spin precession about that field.

Now if we suppose the microstructure length is chosen to be greater than the $L_{\varphi}^{(e)}$, it is possible to 'wash out' the quantum interference related to phase coherence of the 'orbital' part of the wave function retaining at the same time that related to the phase coherence of the spin part and hence, reveal the corresponding conductance oscillations.

Changing the external magnetic field, one can change the 'interference pattern', that is, to control the conductance modulation. We have shown that the strong conductance modulation can be achieved in this way.

\section{Acknowledgements}

One of us (I.T.) is greatly acknowledged to Prof. Robin Nicholas, University of Oxford, UK for discussing the topics touched upon in the paper and his profound comments. 
Fig.1 A sketch of a two-channel semiconductor mesoscopic structure with an additional maghetic field (1) accross one of the channels. On the upper panel $t, t^{\prime}, r, r^{\prime}$ indicate the transmission and reflection matrices at the two junctions $x \leq 0, x \geq L$; $P, P^{\prime}$ stand for the propagation matrices in the middle region $(0 \leq x \leq L)$; 2: the external magnetic field $B_{0}$.

Fig.2 Airy function evaluated by means of the parameters assumed (see the text). $1-\operatorname{Ai}(\mathrm{z})=\operatorname{Ai}\left(\alpha \mathrm{z}_{0}(\mathrm{z}-1)\right), \quad 2-\mathrm{F}(\mathrm{z})=\left(\operatorname{Ai}\left(\alpha \mathrm{z}_{0}(\mathrm{z}-1)\right)\right)^{2}$, where:

$\alpha=\left(\frac{2 m^{*} e E}{\hbar^{2}}+\frac{2 e B_{0} k_{x}}{\hbar c}\right)^{\frac{1}{3}}, \quad z_{0}=\frac{\varepsilon\left(k_{x}\right)-\frac{\hbar^{2} k_{x}^{2}}{2 m^{*}}}{e E+\frac{\hbar e B_{0} k_{x}}{m^{*} c}}, \varepsilon\left(k_{x}\right)-\frac{\hbar^{2} k_{x}^{2}}{2 m^{*}}=\left(\frac{\hbar^{2}}{2 m^{*}}\right)^{\frac{1}{3}}\left[\frac{9 \pi}{8}\left(e E+\frac{\hbar e B_{0} k_{x}}{m^{*} c}\right)\right]^{\frac{2}{3}}$.

Fig.3 Spin phase relaxation time due to Elliot-Yafet mechanism versus external magnetic field for three zinc-blende type semiconductors.

Fig.4 Schematic representation of the precession mechnism of spin relaxation: 1 $S^{2}$-sphere; 2 - a point on the $S^{2}$-sphere corresponds the the initial position of the spin precession axis; 3 - shaded cirlces correspond to the sequential positions of the spin precession axis whose direction changes randomly due to collisions. 


\section{References}

[1] D.D. Awschalom, J.M. Kikkawa, Physics Today 52, 33 (1999)

[2] J.M. Kikkawa, J.A. Gupta, I. Malajovich, D.D. Awschalom, Physica E 9 , 194 (2001)

[3] S. Washburn, R.A. Webb, Adv.in Phys. 35 , 375 (1986)

[4] I.Tralle, J Phys: Condens. Matter 11, 8239 (1999)

[5] Yu.I.Manin, Mathematics and Physics (Ser. Progress in Physics, Ed. by A.Jaffe and D. Ruelle) Birkhäuser, Boston, Stuttgart, 1981 (p.55)

[6] D. Hägle, M. Oestreich, W.W. Rüle, N. Nestle, K.Eberl Appl Phys Lett 73 , 1580 (1998)

[7] K.K. Choi, D.C. Tsui and K. Alavi, Phys Rev B 36 , 7751 (1987)

[8] G. Fishman, G. Lampel, Phys Rev B, 16 , 820 (1977)

[9] F.J. Dyson, Phys Rev 98 , 349 (1955)

[10] D. Bohm, Quantum Theory, (NY: Prentice-Hall Inc. 1952)

[11] V.N. Lisin, B.M. Khabibulin, Fiz Tverd Tel (Sov. Solid State Phys) 17, 1600 (1975)

[12] O.V. Kibis, JETP Lett 66 , 588 (1997)

[13] M. Abramowitz, I.A. Stegun, eds. Handbook of Mathematical Functions , ( Dover Publ. Inc., 1965)

[14] M.I.D'yakonov and V.I.Perel', Fiz. Tverd. Tela, 13,3851 (1981)[Sov. Phys.-Solid State 13, 3023 (1972)]

[15] Yu.A.Bychkov, E.I.Rashba, J.Phys C, 17, 6039 (1984)

[16] V.F.Gantmacher, I.B.Levinson, Carrier scattering in metals and semicoductors, North-Holland, NY-Tokyo, 1987

[17] P.Pfeffer, W.Zawadzki, Phys Rev B, 52, R14 332 (1995)

[18] S. Datta and S. Bandyopadhyay, Phys Rev Lett 58, 717 (1987)

[19] S. Datta, Electronic Transport in Mesoscopic Systems, (Cambridge: Cambr, Univ. Press 1995)

[20] G. Casanova, L'algebre vectorielle , ( Paris: Presses Universitaires de France 1976 ) 
[21] H. Rauch, Europhysics News, 28 , 10 (1997)

[22] J. Lambek, The Mathematical Intelligencer, 17, 7 (1995) 